\title{
Interfacing
}

\section{Interfacing the microcomputer: A component-level approach}

\author{
DAVID T. KRAUSMAN \\ Department of Psychiatry and Behavioral Sciences, Johns Hopkins University School of Medicine, \\ Baltimore, Maryland 21205
}

\begin{abstract}
A method is described for interfacing relatively inexpensive component- and chip-level devices to a standard Intel microcomputer board for providing an on-line physological monitoring system. The system demonstrates basic interfacing techniques and a highly simplified approach for integrating various state-of-the-art peripheral circuit components to a standard microcomputer configuration.
\end{abstract}

The microcomputer has in a few years made the transition from experimental novelty to efficient, costeffective laboratory instrumentation. The development of microprocessors is not the only reason for the extraordinarily rapid growth of this phase of electronic technology. Of at least equal importance is the development of various families of integrated circuits (ICs). Often referred to as "systems on a chip," these IC devices are generally self-contained units on a single 16- or 24-pin chip and include such functional units as analog-to-digital (A/D) converters, digital-to-analog (D/A) converters, clock generators, input-output (I/O) drivers, latches, and so on. Used as peripheral support devices, these miniature systems on chip can be integrated into a basic microcomputer configuration to provide an inexpensive computer system for satisfying a wide variety of applications in medical electronics.

The processing power and usefulness of the microcomputer depends largely on the interfacing circuitry and external peripherals or subsystems connected to it. Interfacing cost considerations are often underestimated or even overlooked when projecting the overall investment in a microcomputer system. Since, as in any computer system, the cost of commercial subsystems or modular interfacing devices may always outweigh by many times the cost of the basic computer itself, the initial low-cost advantage of the microcomputer could be significantly offset if expensive peripherals are required by the system design.

Most commercial peripheral boards provide hardware specifications and features in excess of the usual smallsystem requirements. Therefore, both hardware and cost considerations might be impractical in many applications where these units are integrated into the system. Since ICs are relatively cheap and may be selected with various unique control and conversion functions, it is usually an advantage for the smallsystem designer to build his own peripheral circuits.

\section{MICROCOMPUTERS IN PHYSIOLOGICAL MONITORING}

The need for a low-cost computer-controlled instrument for on-line monitoring and averaging of blood pressure signals initiated the development of the microcomputer system illustrated in the schematic diagram of Figure 1. This system features an inexpensive and straightforward approach to interfacing various chips and discrete components with an Intel singleboard microcomputer. The system provides a practical application useful for physiological or behavioral studies and demonstrates a highly simplified approach to the application of some state-of-the-art interfacing techniques to a basic microcomputer system.

The main component of the micro's peripheral circuitry is a $\$ 12$ National MM 5357 A/D converter chip. This device converts the analog voltage derived from the Statham pressure transducer and associated op-amp circuitry to a digital representation of the pressure value. This unit provides 8 bits of binary resolution (256 parts) with a very high conversion rate of less than 30 microsec. Input analog levels from $-5 \mathrm{~V}$ to $+5 \mathrm{~V}$ produce a full-scale straight binary output from 0 to 255. Although 8 bits is sufficient resolution for this particular application, units of 10 bits $(1,024$ parts $)$ or 12 bits $(4,098)$ provide increased accuracy and would satisfy requirements for more precise measurements.

The physiological monitoring system illustrated in Figure 1 features a heart beat by heart beat simultaneous digital display of systolic blood pressure, diastolic blood pressure, and heart rate (pulse interval projected in beats per minute). These three physiological functions also control corresponding relay and alarm lights that are latched to the "ON" condition if any preset (software) limits are exceeded. A Sonalert beeper permits continuous beat-by-beat audible monitoring 


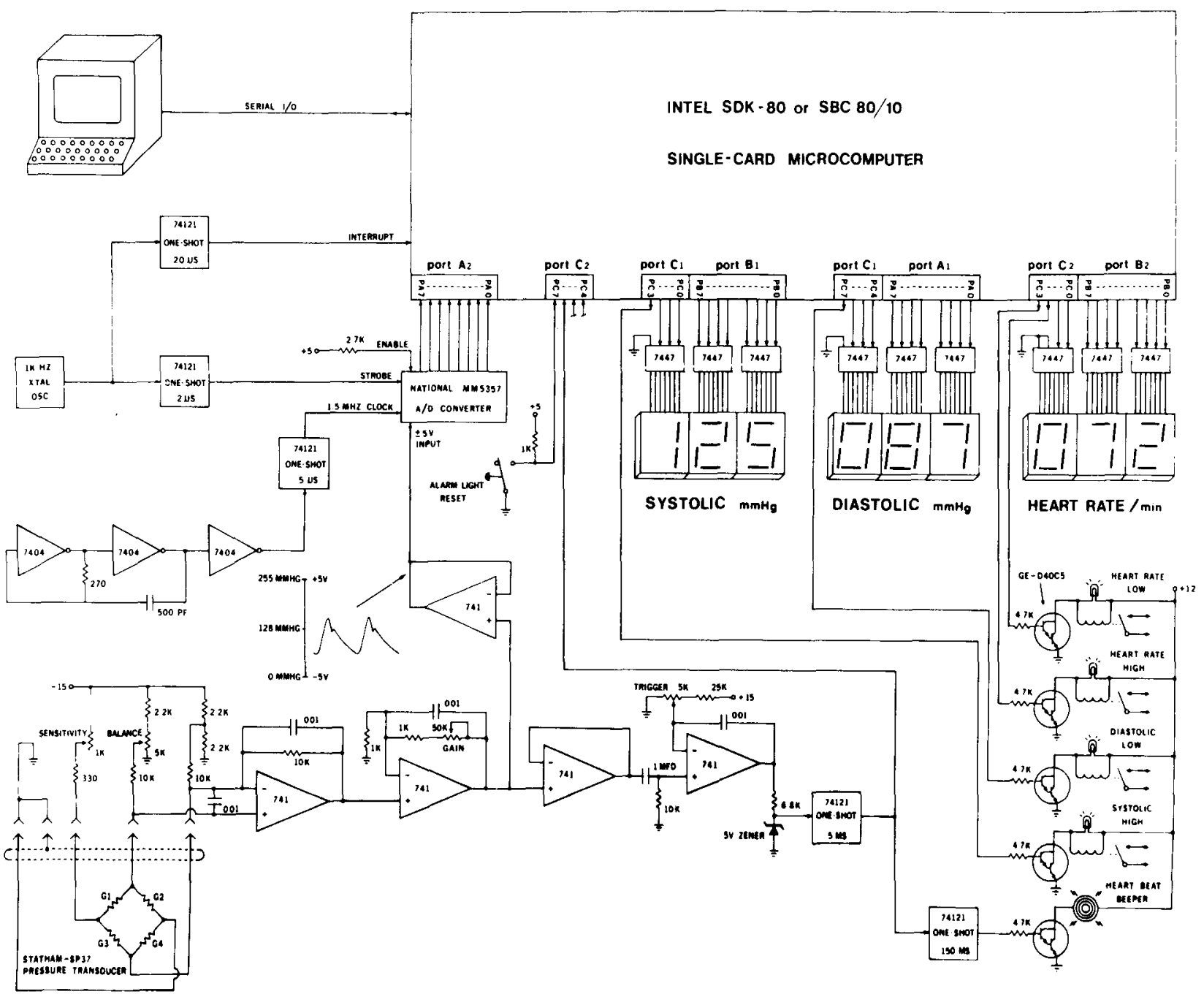

Figure 1. On-line physiological monitoring system features inexpensive ICs and discrete components interfaced to an Intel singleboard microcomputer.

of the pulse rate, and a CRT lists 1 -min averages of the systolic, diastolic, and heart rate values.

The Intel SDK-80 or SBC $80 / 10$ single-card $8080 \mathrm{~A}$ microcomputer system with 48 programmable $\mathrm{I} / \mathrm{O}$ lines, an interrupt capability, and a serial $\mathrm{I} / \mathrm{O}$ port provides an ideal hardware configuration for developing a stand-alone monitoring system. McKenzie (1976) provides a detailed account of the design and architecture characteristics of the 8080 microcomputer system. Krausman (1976) describes some operational features and applications of an 8080 system.

A miniature, unbonded strain-gage blood pressure transducer (Statham Model SP37 or equivalent) attached to the subject at puncture or cannula entry site permits direct measurements of arterial blood pressure (Krausman, 1975). The electrical pressure analog (waveform) derived from the transducer is shifted ("balance" pot) and amplified ("gain" pot) via the 741 op-amps and connected to the input of the 5357 A/D converter (Krausman, 1971). Calibration of the system is accomplished by direct scaling with the op-amp hardware, thereby eliminating cumbersome software algorithms. The pressure range of both systolic and diastolic blood pressure is from 0 to $255 \mathrm{~mm} \mathrm{Hg}(0 \mathrm{~mm} \mathrm{Hg}=-5 \mathrm{~V}, 255 \mathrm{~mm} \mathrm{Hg}=+5 \mathrm{~V})$, which represents the full resolution of the 8-bit A/D converter.

Pulse rate information is derived from the ascending slope of the blood pressure analog. Using a passive RC to differentiate the pressure waveform, the op-amp comparitor triggers ("trigger" pot) on the positive portion of the pressure derivative. This signal drives the audible beeper and provides a heart beat acknowledgement to the microcomputer input port, which is polled during the interrupt service routine.

The $1-\mathrm{kHz}$ crystal oscillator generates a periodic 1 -msec real-time interrupt to serve as the system clock. The clock is used to compute the heart rate value ( 60 per beat period) and to control the timing and I/O functions of the system.

The $1-\mathrm{msec}$ pulse from the crystal oscillator is also used to initiate the conversion strobe to the $A / D$. Using this external strobing technique, handshaking and status polling between the microcomputer and the 
A/D is eliminated. Since the $A / D$ has a conversion rate of less than 30 microsec, the data in the $A / D$ buffer is settled and ready to be transferred with a single input instruction during the interrupt service routine. Although the $\mathrm{A} / \mathrm{D}$ samples at a fixed conversion rate of $1,000 / \mathrm{sec}$, a $200 / \mathrm{sec}$ rate provides sufficient resolution of the pressure waveform. In order to conserve RAM memory, a software divide by 5 allows for a reduced data rate of $200 \mathrm{samples} / \mathrm{sec}$.

Utilizing the programmable $48 \mathrm{I} / \mathrm{O}$ bits contained within the Intel microcomputer, GE Darlington power transistors D40C5 (about 80 cents each) provide a simple drive circuit for the alarm lights and relays. These transistors can also be used to drive solenoids or other control-ty pe devices.

The Intel's I/O ports also facilitate direct connection to a standard 7447 IC driver chip (BCD to seven segment) for driving MAN-1-type light-emitting diode units for displaying systolic and diastolic blood pressures and heart rate functions.

The usual complexities and related high costs of interfacing standard board-level peripherals to a computer system can be significantly reduced if the designer takes advantage of the new chip technology. Laboratory-built $\mathrm{A} / \mathrm{D}, \mathrm{D} / \mathrm{A}, \mathrm{I} / \mathrm{O}$, and other control peripherals can be integrated into microcomputer systems using IC chips and other discrete circuit components for only a small fraction of the cost that would be required if commercial board-level units were used. However, if the designer has a strict time schedule in which the system must be completed and the budget allows for additional expenditures ranging from $\$ 250$ to $\$ 800$, the board-level interfacing approach is appropriate.

The device described is an example of an application where either approach could be justified. A Burroughs SSD1000-0041 self-scan display panel (\$250) and a Burr-Brown MP8608 A/D board (\$405) are two commercial peripheral units that would have saved considerable wiring, development, and programming time if they had been integrated into the system. Since cost consideration was the most important restriction in the development of the monitoring system, the lab-built National A/D circuit and the MAN-1 lightemitting diode display units for a combined cost of less than $\$ 30$ was the obvious choice for the described system.

\section{REFERENCES}

Krausman, D. T. Transducer bridge amplifier to directly drive low sensitivity oscillographic recorders. Psychophysiology. 1971, 7, 279-280.

Krausman, D. T. Methods and procedure for monitoring and recording blood pressure. American Psychologist, 1975, 30, 285-294.

Krausman, D. T. The microcomputer kit: An excellent small system development tool. Behavior Research Methods \& Instrumentation, 1976, 8, 501-507.

McKenzie, K. A structured approach to microcomputer system design. Behavior Research Methods \& Instrumentation, 1976, 8. 123-128.

(Received for publication May 25, 1978; revision accepted June 23,1978 .) 